\title{
Epigenetic control of cancer by neuropeptides (Review)
}

\author{
KARINA GALOIAN and PARTHIK PATEL \\ Department of Orthopedics, Miller School of Medicine, University of Miami, Miami, FL 33136, USA
}

Received September 8, 2016; Accepted October 26, 2016

DOI: $10.3892 /$ br.2016.804

\begin{abstract}
Neuropeptides act as neurohormones, neurotransmitters and/or neuromodulators. Neuropeptides maintain physiological homeostasis and are paramount in molecular mechanisms of disease progression and regulation, including in cancer. Neuropeptides, by their definition, originate and are secreted from the neuronal cells, they are able to signal to neighboring cells or are released into the blood flow, if they act as neurohormones. The majority of neuropeptides exert their functions through $\mathrm{G}$ protein-coupled receptors, with certain exceptions. Although previous studies indicate that neuropeptides function in supporting proliferation of malignant cells in many types of solid tumor, the antitumorigenic action of the neuropeptides and their receptors, for example, in gastric cancers and chondrosarcoma, were also reported. It is known that epigenetically modified chromatin regulates molecular mechanisms involved in gene expression and malignant progression. The epigenetic modifications are genetically heritable, although they do not cause changes in DNA sequence. DNA methylation, histone modifications and miRNA expression are subject to those modifications. While there is substantial data on epigenetic regulation of neuropeptides, the epigenetic control of cancer by neuropeptides is considered to be uncharted territory. The aim of the current review is to describe the involvement of neuropeptides in the epigenetic machinery of cancer based on data obtained from our laboratory and from other authors.
\end{abstract}

\section{Contents}

1. Introduction

2. Neuropeptides involved in epigenetic control of cancer

3. Conclusion

Correspondence to: Professor Karina Galoian, Department of Orthopedics, Miller School of Medicine, University of Miami, 1600 NW 10th Avenue, Miami, FL 33136, USA

E-mail:kgaloian@med.miami.edu

Key words: cancer, neuropeptides, epigenetics, proline-rich polypeptide-1, neurotensin, vasoactive intestinal peptide, pituitary adenylate cyclase-activating polypeptide, gastrin

\section{Introduction}

Neuropeptides are an important class of messenger molecules that carry information between neurons; they can act as neurohormones, neurotransmitters, and/or neuromodulators, maintain physiological homeostasis and are involved in regulation of malignant disease progression. Notably, peptide hormones and neuropeptides are synthesized by the same route and enzymes from common precursor cleavage, resulting in the production of bioactive peptides. The majority of neuropeptides exert their functions through $G$ protein-coupled receptors, with certain exceptions (1). The predominant notion was that neuropeptides possess protumorigenic functions demonstrated via paracrine and autocrine loops of regulation, which were reported in different types of solid tumors (2-6); however, antitumorigenic action of the neuropeptides and their receptors has been reported for gastric cancers (6), chondrosarcoma (7-9) and triple negative breast cancer (10). The epigenetic modifications are crucial in regulating gene expression and control the progression of cancer. The epigenetic modifications are genetically heritable, but do not cause changes in DNA sequence. DNA methylation, histone modifications and miRNA expression are subject to those modifications. While there is substantial data regarding epigenetic regulation of neuropeptides (11), the epigenetic control of cancer by neuropeptides is considered to be a particularly promising discipline and requires close attention and further development.

The current review aims to present the involvement of certain neuropeptides in the epigenetic machinery of cancer (12-19) based upon data obtained from our laboratory and from other authors.

\section{Neuropeptides involved in epigenetic control of cancer}

Proline-rich polypeptide-1 (PRP-1; galarmin). PRP-1 is a cytokine hypothalamic neuropeptide produced by neurosecretory granules of neurohypophysis, in Nucleus supraopticus and Nucleus paraventricularis, the same neurons from which vasopressin and oxytocin originate. PRP-1 is derived from its precursor, neurophysin-vasopressin-associated glycoprotein precursor (20). In addition, large quantities of PRP-1 were detected in bone marrow granulocytes (21). The antimicrobial, antitumorigenic and antineurodegenerative properties for this immunomodulatory cytokine were previously reported (22) and the quantification of hypothalamic PRP-1 in the blood was recently documented (23). Furthermore, our prevous studies described that PRP-1, mammalian target of rapamycin 
complex 1 inhibitor, exerts antiproliferative cytostatic effects in chondrosarcoma $(7-9,24)$ and breast cancer cells $(10)$.

Chondrosarcoma is a type of cartilage cancer, which does not respond to chemotherapy or radiation, and eventually metastasizes rendering surgery as the only treatment option; thus, the search for novel therapeutic strategies is considered to be relevant and urgent (25).

Although PRP-1 is a neuropeptide, our preliminary results (not yet published) did not indicate that PRP-1 exerts its actions via the G protein-coupled receptor. Cytostatic PRP-1 manifests its antiproliferative effect via cell cycle regulation in cancer (10), as well as by its unique ability to upregulate tumor suppressor proteins (26) and micro RNA (miR) tumor suppressors (miR20a, miR125b and miR192) while causing downregulation of onco-miRs (miR509-3p, miR589, miR490-3p and miR 550) in the human chondrosarcoma cell line, JJ012 (27), demonstrating the possibility for future therapeutic interventions.

PRP-1 epigenetically regulates embryonic stem cell marker, miRNA 302c (miR302C) and its targets. miR302C, a component of the miR302 367 cluster, was significantly downregulated by PRP-1. Notably, this cluster is not expressed in adult mesenchymal stem cells and normal tissue (28), although it is particularly characteristic, with a significant role in tumors and human embryonic stem cells, where it regulates renewal of stem cells and the process of differentiation (29). This may explain the strong tumor growth inhibitory role of PRP-1 in certain tumors, particularly in chondrosarcomas and multipotent adult stem cells (marrow-isolated adult multilineage inducible cells) of embryonic primitive type $(28,30)$. Our collaborative study of glioblastoma demonstrated that PRP-1 did not exert inhibitory action on the growth of this type of tumor, as in glioblastoma this cluster suppresses stemness $(28,29)$. This fact served as the basis for the paradigm that the antiproliferative action of PRP-1 is defined by the presence of miR302C and its stemness-inducing potential in particular types of tumor.

The stemness markers, targets of the miR302C polycomb protein, BMI-1, NANOG (28) and c-Myc $(8,24,28)$, were markedly downregulated by PRP-1 (28). Interestingly, PRP-1 demonstrated the inhibition of giant cell tumors of the bone and, conversely, supported the growth of human normal bone marrow stromal cells (31). Epigenetic regulation of miR302C expression (32) is defined by positive regulatory activity of JMJD2, H3K9me2 demethylase and NANOG in the promoter regions (28). Our previous data indicated that PRP-1 inhibited H3K9 demethylase activity (JMJD1 and JMJD2) leading to the conclusion that miR302C activity suppression by this neuropeptide is epigenetically regulated (28).

Vasoactive intestinal peptide (VIP) and pituitary adenylate cyclase activating polypeptide (PACAP). VIP is produced by various cells, however, its primary location is within neurons; this peptide is expressed in the peripheral and central nervous systems, as well as in certain tumors (3,33-36). In a previous study, VIP receptor antagonists facilitated chemotherapeutic agents to cause apoptosis in certain cancer cell lines (33); VIP is cell context-dependent and is known to contribute to leukemogenesis (34). There is reported similarity of VIP with pituitary adenylate cyclase, which belongs to the secretin family. PACAP binds to the G protein-coupled receptor and belongs to the secretin glucagon VIP peptide group (35).

VIP shares $68 \%$ homology with PACAP, and is considered to be a rational target against inflammatory disease (35). Notable data on transcriptional modulation of genes involved in production of inflammatory products, such as inducible nitric oxide synthases, revealed the inhibitory effect of VIP on gene transcription, which may possess marked therapeutic potential preventing production of inflammatory molecules (37).

PACAP is significantly involved in regulating the immune response, and is found in neurons and the immune system. PACAP is comprised of three known receptors, PAC-1, VPAC1 (3) and VPAC2, and is involved in signal transduction pathways and, depending on the tumor type, either directly suppresses or promotes tumor growth (36). In cervical cancer, PACAP is considered as a methylation biomarker for cervical cancer early detection $(38,39)$. In a previous study, a low expression of PACAP was demonstrated in cervical cancer, which was due to hypermethylation in its promoter and was correlated with tumor development. Treatment with 5'-Aza-2', a methyltransferase inhibitor, or with histone deacetylase inhibitor (HDACi), trichostatin reactivated PACAP gene expression (38). PACAP in humans is encoded by adenylate cyclase-activating polypeptide 1 (ADCYAP1). Another study reported that the methylation of ADCYAP1 may be highly associated with the development of cervical cancer and that gene promoter hypermethylation suppressed gene expression (40).

Gastrin. Gastrin-releasing peptide (GRP) is a human neuropeptide that controls gastrin release and regulates gastric acid production. Gastrin is released from $\mathrm{G}$ cells as a result of vagus nerve postganglionic fiber innervation of stomach $G$ cells. GRP is engaged in stress regulation of the biological circadian rhythm sending signals to the hypothalamic suprachiasmatic nuclei $(41,42)$. GRP and neuromedin $C$ are important in various types of cancer (42). It was established that pro-GRP is produced in small lung cancer cells and is considered to be a biomarker (43). The peptide is utilized for therapeutic purposes, measuring the efficacy of chemo- and radiation therapies. In normal conditions GRP is expressed in the bronchial epithelium and promotes lung development at the fetal stage. When it is associated with bombesin (BB)-like peptide receptors (44), such as GRP receptor (GRPR), it predominantely (although with certain exceptions) acts as a growth promoter for small cell lung cancer. Conversely, epithelial cells of the adult colon under normal conditions are deprived of GRP receptor expression. Notably, GRP/GRPR expression in tumors was identified to correlate with improved patient survival rates and reduced metastatic spread. However, the mechanisms involved in these manifestations require further investigation $(45,46)$. Previous findings provide evidence that a GRPR antagonist stimulates the growth of cancer cells, and that the stimulatory effects were prevented by the HDACi, suggesting that GRPR may interact with epigenetic mechanisms in regulating neuroblastoma cell growth. However, 100 nM GRPR stimulated proliferation of Neuro-2a murine neuroblastoma cells in vitro; the stimulatory effects were prevented by the HDACi (47). Involvement of BB in the acetylation of the androgen receptor and activation of 
Table I. Epigenetic regulation of neuropeptides in cancer.

Author, year

Description

Refs.

Misawa et al, 2014

Misawa et al, 2016

Misawa et al, 2015

David et al, 2009

Misawa et al, 2013

Mori et al, 2006

Kamimae et al, 2015

Zhong et al, 2007
CpG hypermethylation was reported as the silencing mechanism for the neuropeptide, GAL and its receptor, GALR1/2 gene, leading to inactivation of their tumor suppressing properties in HNSCC resulting in tumorigenesis. Therefore, the gene's methylation status was proposed as an important biomarker for clinical outcome

CpG hypermethylation was attributed as a possible mechanism for SST and SSTR1 methylation profiles for HNSCC tumorigenesis

TAC1 is the precursor protein for neuroendocrine peptides, including substance $\mathrm{P}$, and is centrally involved in gastric secretion, motility, mucosal immunity and cell proliferation. The authors indicated the aberrant silencing of TAC1 in GC by promoter hypermethylation The specific mechanisms, elucidated by the authors, of TAC1 and TACR 1 gene inactivation via frequent promoter hypermethylation methylation led to tumorigenesis Gene silencing via promoter hypermethylation of SST and TAC1 and 5, leading to colon cancer tumorigenesis was highlighted in this study

NTSR1 methylation was associated with lateral and non-invasive growth of colorectal tumors, while low levels of methylation contributed to the malignant potential via activation of NTSR 1 and the clinical implications were documented

In this study, oxytocin receptor was dominantly regulated by histone deacetylation and demonstrated to be frequently downregulated in lung tumors
$(61,62)$

CpG, cytosine-guanine dinucleotide; GAL, galanin; HNSCC, head and neck squamous cell carcinoma; SST, somatostatin; SSTR1, somatostatin receptor type 1; TAC1, tachykinin-1; GC, gastric cancer; TACR1, tachykinin receptor type 1; NTSR1, neurotensin receptor 1.

androgen-associated genes in prostate cancer cells via activation of p300 histone acetyltransferase activity was reported in another study (48). The effects of a BB/GRP receptor antagonist, PD176252, and HDACi, MS-275, were investigated in human lung cancer cell lines and the results indicated the ability of GRPR antagonists to potentiate the action of HDACi on lung cancer cellular proliferation by increasing the expression of tumor suppressor genes (49).

Gastrin regulates heterochromatin protein 1 (HP1) expression in cancer and HP1 is directly involved in epigenetic control of gene transcription. The methylation of histone $\mathrm{H} 3$ at lysine 9 along with HP1 recruitment secures chromatin assembly to epigenetically control the genome (50). Experimental evidence indicates that blocking GRP signaling results in the downregulation of $\mathrm{HP}^{\mathrm{Hs} \beta}$ expression, resulting in colon cancer cell invasiveness (45). ChIP-seq revealed the targets of HP1 $\beta$ due to BB/GRPR activation, which were as follows: Interleukin-1 receptor accessory protein like 2 , family with sequence similarity 13 member A, 1,4- $\alpha$-glucan branching enzyme 1, polo like kinase 3, and solute carrier organic anion transporter family member 1B3 (51). In addition, gastrin induced the overexpression of miR222 that led to cytoplasmic mislocalisation of p27kip1, causing cell migration. Data indicated a novel mechanism that involves gastrin, which is associated with tumor development. Notably, miR222 may serve as a promising biomarker for observing gastrin-induced premalignant changes (52). The role of GRPR is reported to be tumorigenic. Therefore, the silencing of GRPR suppresses tumorigenesis and the metastatic potential of neuroblastoma (53). Overexpression of miR-335 and miR-363 decreased tumorigenicity as measured by clonogenicity, anchorage-independent growth, and metastasis determined by cell invasion assay and liver metastasis in vivo. Thus, miR-335 and miR-363 functioned as tumor suppressors in GRPR-silenced neuroblastoma (54). Novel therapeutic strategies against aggressive neuroblastoma may be derived from upregulation of miR-335 and miR-363, which are capable of reversing tumorigenicity and blocking cell transformation. Initiation of antral gastric cancer was associated with epigenetic silencing of trefoil factor 1 (TFF1) tumor suppressor gene silencing By contrast, inhibition of gastric carcinogenesis by the hormone gastrin was mediated by suppression of TFF1 epigenetic silencing (6).

Somatostatin (SST) is a gut peptide that is able to inhibit the growth of tumor cells in gastric cancer, inhibit gastrin release, gastric acid production, and is regarded as a novel cancer repressive polypeptide. SST promoter methylation is a common occurrence in human gastric cancer, and is connected with a decrease in SST protein and RNA levels, as well as being associated with gastric carcinogens. A significant increase in SST promoter was identified in tumor samples when compared with healthy samples. Thus, the promoter DNA methylation was defined as an epigenetic mechanism of SST expression regulation. SST is therefore a potential biomarker for gastric cancers (55).

Neurotensin (NTS). NTS is a neuropeptide that is involved in the regulation of luteinizing hormone and it is connected to the dopaminergic system. It is highly present in the hypothalamic nucleus accumbens and amygdala, inducing multiple effects, such as hypothermal and increased locomotor activity, and smooth muscle contruction in the small intestine (56). NTS is considered to be a cancer promoting mitogen in colon cancer (57). A notable connection between its neurotensin receptor 1 (NTR1) and induction of inflammation, and 
tumor growth mediated by upregulated expression of certain miRNAs (such as miR21 and miR155) has been reported (58). Their upregulation in human colon cancer, also caused downregulation of major tumor suppressors, including phosphatase and tensin homolog and suppressor of cytokine signaling proteins (58).

NTS and its receptor are implicated in cancer progression. The upregulation of miR-29b-1 and miR-129-3p expression led to impaired proliferation of glioma cells. NTS signaling upregulated c-Myc production and inhibited the above-mentioned miRNAs. These results indicate that the NTS/NTSR1/c-Myc/miRNA axis is important in the pathogenesis of glioblastoma and may be considered as a potential therapeutic target (59).

\section{Conclusion}

Epigenetics in cancer research is a rapidly developing field. An important lesson is obvious; that the inactivation of tumor suppression pathways in cancer presents potential therapeutic opportunities for epigenetic therapy intervention (60). To better understand the mechanism of derailed epigenetic regulation in malignancies, the insight into epigenetic control in normal and embryonic tissues is of utmost importance. Thus, epigenetic regulation of cancer by neuropeptides is an exciting and novel direction; however, it is in its infancy, although there is substantial data on epigenetic regulation of neuropeptides, some examples are shown in Table I (61-68). Certain neuropeptides, being natural compounds by their origin, possess powerful antitumorigenic, tumor suppressor and immunomodulatory properties, providing added benefit for future potential therapeutic strategies. Whether they are cytotoxic or cytostatic by nature, they are promising in the battle against cancer.

\section{Acknowledgements}

The current study was supported in part by a gift from the Ratcliffe Foundation to Miami Center of Orthopedic Research and Education.

\section{References}

1. Fricker LD: Neuropeptides and Other Bioactive Peptides: From Discovery to Function. Morgan \& Claypool Life Sciences, San Rafael, CA, p118, 2012.

2. Rozengurt E: Neuropeptides as growth factors for normal and cancerous cells. Trends Endocrinol Metab 13: 128-134, 2002

3. Liu S, Zeng Y, Li Y, Guo W, Liu J and Ouyang N: VPAC1 overexpression is associated with poor differentiation in colon cancer. Tumour Biol 35: 6397-6404, 2014.

4. Evers BM, Parekh D, Townsend CM Jr and Thompson JC: Somatostatin and analogues in the treatment of cancer. A review. Ann Surg 213: 190-198, 1991.

5. Reubi JC, Gugger M, Waser B and Schaer JCY: Y(1)-mediated effect of neuropeptide $\mathrm{Y}$ in cancer: Breast carcinomas as targets. Cancer Res 61: 4636-4641, 2001.

6. Tomita H, Takaishi S, Menheniott TR, Yang X, Shibata W, Jin G, Betz KS, Kawakami K, Minamoto T and Tomasetto C: Inhibition of gastric carcinogenesis by the hormone gastrin is mediated by suppression of TFF1 epigenetic silencing. Gastroenterology 140: 879-891, 2011.

7. Galoian K, Temple HT and Galoyan A: mTORC1 inhibition and ECM-cell adhesion-independent drug resistance via PI3K-AKT and PI3K-RAS-MAPK feedback loops. Tumour Biol 33: 885-890, 2012.
8. Galoian K, Scully S and Galoyan A: Myc-oncogene inactivating effect by proline rich polypeptide (PRP-1) in chondrosarcoma JJ012 cells. Neurochem Res 34: 379-385, 2009.

9. Galoian K, Scully S, McNamara G, Flynn P and Galoyan A: Antitumorigenic effect of brain proline rich polypeptide-1 in human chondrosarcoma. Neurochem Res 34: 2117-2121, 2009.

10. Galoian KA, Temple TH and Galoyan A: Cytostatic effect of novel mTOR inhibitor, PRP-1 (galarmin) in MDA 231 (ER-) breast carcinoma cell line. PRP-1 inhibits mesenchymal tumors. Tumour Biol 32: 745-751, 2011.

11. Zhang $X$ and Ho SM: Epigenetics meets endocrinology. J Mol Endocrinol 46: R11-R32, 2011.

12. Kulis M and Esteller M: DNA methylation and cancer. Adv Genet 70: 27-56, 2010.

13. Klose RJ, Kallin EM and Zhang Y: JmjC-domain-containing proteins and histone demethylation. Nat Rev Genet 7: 715-727, 2006.

14. Bannister AJ, Zegerman P, Partridge JF, Miska EA, Thomas JO, Allshire RC and Kouzarides T: Selective recognition of methylated lysine 9 on histone H3 by the HP1 chromo domain. Nature 410: 120-124, 2001.

15. Shi Y, Lan F, Matson C, Mulligan P, Whetstine JR, Cole PA, Casero RA and Shi Y: Histone demethylation mediated by the nuclear amine oxidase homolog LSD1. Cell 119: 941-953, 2004.

16. Whetstine JR, Nottke A, Lan F, Huarte M, Smolikov S, Chen Z, Spooner E, Li E, Zhang G, Colaiacovo M, et al: Reversal of histone lysine trimethylation by the JMJD2 family of histone demethylases. Cell 125: 467-481, 2006.

17. Klose RJ, Yamane K, Bae Y, Zhang D, Erdjument-Bromage H, Tempst P, Wong J and Zhang Y: The transcriptional repressor JHDM3A demethylates trimethyl histone H3 lysine 9 and lysine 36. Nature 442: 312-316, 2006.

18. Sato F, Tsuchiya S, Meltzer SJ and Shimizu K: MicroRNAs and epigenetics. FEBS J 278: 1598-1609, 2011.

19. Subramanian S and Kartha RV: MicroRNA-mediated gene regulations in human sarcomas. Cell Mol Life Sci 69: 3571-3585, 2012.

20. Galoyan A: Neurochemistry of brain neuroendocrine immune system: Signal molecules. Neurochem Res 25: 1343-1355, 2000.

21. Galoyan AA, Korochkin LI, Rybalkina EJ, Pavlova GV, Saburina IN, Zaraiski EI, Galoyan NA, Davtyan TK, Bezirganyan KB and Revishchin AV: Hypothalamic proline-rich polypeptide enhances bone marrow colony-forming cell proliferation and stromal progenitor cell differentiation. Cell Transplant 17: 1061-1066, 2008.

22. Galoyan A: Concepts of neuroendocrine cardiology and neuroendocrine immunology, chemistry and biology of signal molecules. Neurochem Res 35: 2001-2017, 2010.

23. Abrahamyan SS, Davtyan TK, Khachatryan AR, Tumasyan NV, Sahakyan IK, Harutyunyan HA, Chailyan SG and Galoyan A: Quantification of the hypothalamic proline rich polypeptide-1 in rat blood serum. Neurochem J 8: 38-43, 2014.

24. Galoian K, Temple TH and Galoyan A: Cytostatic effect of the hypothalamic cytokine PRP-1 is mediated by mTOR and cMyc inhibition in high grade chondrosarcoma. Neurochem Res 36: 812-818, 2011.

25. Ozaki T, Hillmann A, Lindner N, Blasius S and Winkelmann W: Metastasis of chondrosarcoma. J Cancer Res Clin Oncol 122: 625-628, 1996.

26. Galoian K, Qureshi A, Wideroff G and Temple HT: Restoration of desmosomal junction protein expression and inhibition of H3K9-specific histone demethylase activity by cytostatic proline-rich polypeptide-1 leads to suppression of tumorigenic potential in human chondrosarcoma cells. Mol Clin Oncol 3: 171-178, 2015.

27. Galoian KA, Guettouche T, Issac B, Qureshi A and Temple HT: Regulation of onco and tumor suppressor MiRNAs by mTORC1 inhibitor PRP-1 in human chondrosarcoma. Tumour Biol 35: 2335-2341, 2014.

28. Galoian K, Qureshi A, D'Ippolito G, Schiller PC, Molinari M, Johnstone AL, Brothers SP, Paz AC and Temple HT: Epigenetic regulation of embryonic stem cell marker miR302C in human chondrosarcoma as determinant of antiproliferative activity of proline-rich polypeptide 1. Int J Oncol 47: 465-472, 2015.

29. Zhang B, Pan $X$ and Anderson TA: MicroRNA: A new player in stem cells. J Cell Physiol 209: 266-269, 2006.

30. D'Ippolito G, Diabira S, Howard GA, Menei P, Roos BA and Schiller PC: Marrow-isolated adult multilineage inducible (MIAMI) cells, a unique population of postnatal young and old human cells with extensive expansion and differentiation potential. J Cell Sci 117: 2971-2981, 2004. 
31. Chailakhyan RK, Gerasimov YV, Chailakhyan MR and Galoyan AA: Proline-rich hypothalamic polypeptide has opposite effects on the proliferation of human normal bone marrow stromal cells and human giant-cell tumour stromal cells Neurochem Res 35: 934-939, 2010.

32. Hadjimichael C, Chanoumidou K, Papadopoulou N, Arampatzi P, Papamatheakis J and Kretsovali A: Common stemness regulators of embryonic and cancer stem cells. World J Stem Cells 7 : $1150-1184,2015$

33. Moody TW and Gozes I: Vasoactive intestinal peptide receptors: A molecular target in breast and lung cancer. Curr Pharm Des 13: 1099-1104, 2007.

34. Dorsam GP, Benton K, Failing J and Batra S: Vasoactive intestinal peptide signaling axis in human leukemia. World J Biol Chem 2: 146-160, 2011

35. Wang HY, Jiang XM and Ganea DJ: The neuropeptides VIP and PACAP inhibit IL-2 transcription by decreasing cJun and increasing JunB expression in T cells. J Neuroimmunol 104 $68-78,2000$

36. Balster DA, O'Dorisio MS, Albers AR, Park SK and Qualman SJ: Suppression of tumorigenicity in neuroblastoma cells by upregulation of human vasoactive intestinal peptide receptor type 1 Regul Pept 109: 155-165, 2002.

37. Ibrahim H, Barrow P and Foster N: Transcriptional modulation by VIP: A rational target against inflammatory disease. Clin Epigenetics 2: 213-222, 2011.

38. Lee JH, Lee JY, Rho SB, Choi JS, Lee DG, An S, Oh T, Choi DC and Lee SH: PACAP inhibits tumor growth and interferes with clusterin in cervical carcinomas. FEBS Lett 588: 4730-4739, 2014.

39. Jung S, Yi L, Jeong D, Kim J, An S, Oh TJ, Kim CH, Kim CJ, Yang Y, Kim KI, et al: The role of ADCYAP1, adenylate cyclase activating polypeptide 1, as a methylation biomarker for the early detection of cervical cancer. Oncol Rep 25: 245-252, 2011.

40. Ki EY, Lee KH, Hur SY, Rhee JE, Kee MK, Kang C and Park JS Methylation of Cervical Neoplastic Cells Infected With Human Papillomavirus 16. Int J Gynecol Cancer 26: 176-183, 2016.

41. Merali Z, McIntosh J and Anisman H: Role of bombesin-related peptides in the control of food intake. Neuropeptides 33: 376-386, 1999.

42. Uchida K, Kojima A, Morokawa N, Tanabe O, Anzai C, Kawakami M, Eto Y and Yoshimura K: Expression of progastrin-releasing peptide and gastrin-releasing peptide receptor mRNA transcripts in tumor cells of patients with small cell lung cancer. J Cancer Res Clin Oncol 128: 633-640, 2002

43. Miyake Y, Kodama T and Yamaguchi K: Pro-gastrin-releasing peptide(31-98) is a specific tumor marker in patients with smal cell lung carcinoma. Cancer Res 54: 2136-2140, 1994.

44. Begum AA, Moyle PM and Toth I: Investigation of bombesin peptide as a targeting ligand for the gastrin releasing peptide (GRP) receptor. Bioorg Med Chem 25: 5834-5841, 2016.

45. Tell R, Rivera CA, Eskra J, Taglia LN, Blunier A, Wang QT and Benya RV: Gastrin-releasing peptide signaling alters colon cancer invasiveness via heterochromatin protein $1 \mathrm{Hs} \beta$. Am J Pathol 178: 672-678, 2011.

46. Rivera CA, Ahlberg NC, Taglia L, Kumar M, Blunier A and Benya RV: Expression of GRP and its receptor is associated with improved survival in patients with colon cancer. Clin Exp Metastasis 26: 663-671, 2009.

47. Abujamra AL, Almeida VR, Brunetto AL, Schwartsmann G and Roesler R: A gastrin-releasing peptide receptor antagonis stimulates Neuro2a neuroblastoma cell growth: Prevention by a histone deacetylase inhibitor. Cell Biol Int 33: 899-903, 2009.

48. Gong J, Zhu J, Goodman OB Jr, Pestell RG, Schlegel PN, Nanus DM and Shen R: Activation of p300 histone acetyltransferase activity and acetylation of the androgen receptor by bombesin in prostate cancer cells. Oncogene 25: 2011-2021, 2006

49. Moody TW, Nakagawa T, Kang Y, Jakowlew S, Chan D and Jensen RT: Bombesin/gastrin-releasing peptide receptor antagonists increase the ability of histone deacetylase inhibitors to reduce lung cancer proliferation. J Mol Neurosci 28: 231-238, 2006

50. Grewal SI and Jia S: Heterochromatin revisited. Nat Rev Genet 8: $35-46,2007$.
51. Tell R, Wang QT, Blunier A and Benya RV: Identification of ChIP-seq mapped targets of HP1 $\beta$ due to bombesin/GRP receptor activation. Clin Epigenetics 2: 331-338, 2011

52. Lloyd KA, Moore AR, Parsons BN, O'Hara A, Boyce M, Dockray GJ, Varro A and Pritchard DM: Gastrin-induced miR-222 promotes gastric tumor development by suppressing p27kip1. Oncotarget: Jun 14, 2016 (Epub ahead of print).

53. Qiao J, Kang J, Ishola TA, Rychahou PG, Evers BM and Chung DH: Gastrin-releasing peptide receptor silencing suppresses the tumorigenesis and metastatic potential of neuroblastoma. Proc Natl Acad Sci USA 105: 12891-12896, 2008.

54. Qiao J, Lee S, Paul P, Theiss L, Tiao J, Qiao L, Kong A and Chung DH: miR-335 and miR-363 regulation of neuroblastoma tumorigenesis and metastasis. Surgery 154: 226-233, 2013.

55. Jackson K, Soutto M, Peng D, Hu T, Marshal D and El-Rifai W: Epigenetic silencing of somatostatin in gastric cancer. Dig Dis Sci 56: 125-130, 2011

56. Friry C, Feliciangeli S, Richard F, Kitabgi P and Rovere C: Production of recombinant large proneurotensin/neuromedin $\mathrm{N}$-derived peptides and characterization of their binding and biological activity. Biochem Biophys Res Commun 290: 1161-1168, 2002.

57. Wang X, Wang Q, Ives KL and Evers BM: Curcumin inhibits neurotensin-mediated interleukin-8 production and migration of HCT116 human colon cancer cells. Clin Cancer Res 12: 5346-5355, 2006.

58. Bakirtzi K, Hatziapostolou M, Karagiannides I, Polytarchou C, Jaeger S, Iliopoulos D and Pothoulakis C: Neurotensin signaling activates microRNAs-21 and -155 and Akt, promotes tumor growth in mice, and is increased in human colon tumors. Gastroenterology 141: 1749-61.e1, 2011.

59. Ouyang Q, Chen G, Zhou J, Li L, Dong Z, Yang R, Xu L, $\mathrm{Cui} \mathrm{H}, \mathrm{Xu} \mathrm{M}$ and $\mathrm{Yi}$ L: Neurotensin signaling stimulates glioblastoma cell proliferation by upregulating c-Myc and inhibiting miR-29b-1 and miR-129-3p. Neuro-oncol 18: 216-226, 2016.

60. Lund AH and van Lohuizen M: Epigenetics and cancer. Genes Dev 18: 2315-2335, 2004.

61. Misawa K, Misawa Y, Kanazawa T, Mochizuki D, Imai A, Endo S, Carey TE and Mineta H: Epigenetic inactivation of galanin and GALR1/2 is associated with early recurrence in head and neck cancer. Clin Exp Metastasis 33: 187-195, 2016.

62. Misawa Y, Misawa K, Kanazawa T, Uehara T, Endo S, Mochizuki D, Yamatodani T, Carey TE and Mineta H: Tumor suppressor activity and inactivation of galanin receptor type 2 by aberrant promoter methylation in head and neck cancer. Cancer 120: 205-213, 2014.

63. Misawa K, Misawa Y, Kondo H, Mochizuki D, Imai A, Fukushima H, Uehara T, Kanazawa T and Mineta H: Aberrant methylation inactivates somatostatin and somatostatin receptor type 1 in head and neck squamous cell carcinoma. PLoS One 10: e0118588, 2015.

64. David S, Kan T, Cheng Y, Agarwal R, Jin Z and Mori Y: Aberrant silencing of the endocrine peptide gene tachykinin-1 in gastric cancer. Biochem Biophys Res Commun 378: 605-609, 2009.

65. Misawa K, Kanazawa T, Misawa Y, Imai A, Uehara T, Mochizuki D, Endo S, Takahashi G and Mineta H: Frequent promoter hypermethylation of tachykinin-1 and tachykinin receptor type 1 is a potential biomarker for head and neck cancer. J Cancer Res Clin Oncol 139: 879-889, 2013.

66. Mori Y, Cai K, Cheng Y, Wang S, Paun B, Hamilton JP, Jin Z, Sato F, Berki AT, Kan T, et al: A genome-wide search identifies epigenetic silencing of somatostatin, tachykinin-1, and 5 other genes in colon cancer. Gastroenterology 131: 797-808, 2006.

67. Kamimae S, Yamamoto E, Kai M, Niinuma T, Yamano HO, Nojima M, Yoshikawa K, Kimura T, Takagi R, Harada E, et al: Epigenetic silencing of NTSR 1 is associated with lateral and noninvasive growth of colorectal tumors. Oncotarget 6: 29975-29990, 2015 .

68. Zhong S, Fields CR, Su N, Pan YX and Robertson KD: Pharmacologic inhibition of epigenetic modifications, coupled with gene expression profiling, reveals novel targets of aberrant DNA methylation and histone deacetylation in lung cancer. Oncogene 26: 2621-2634, 2007. 